\title{
A RELATION BETWEEN OBSTRUCTIONS AND FUNCTIONAL COHOMOLOGY OPERATIONS
}

\author{
ROBERT D. LITTLE
}

\begin{abstract}
In a recent paper, $P$. Olum developed a formula which relates the obstruction to an extension and the induced homomorphism in cohomology. In the present paper, we develop an analogous formula which relates the obstruction to an extension and a certain functional cohomology operation. This operation is a generalization of the standard functional cohomology operation of Steenrod. Olum showed that his formula is particularly useful when the range space of the extension problem is one of the classifying spaces $\mathrm{BU}, \mathrm{BO}$, or BSp. Our formula is also useful in this context and we show that it can be used to compute the obstruction in certain situations where the Olum formula fails.
\end{abstract}

1. Introduction. In [7], Olum develops a formula which relates an obstruction in an arbitrary dimension to the induced homomorphism of cohomology groups. In this paper, we state an analogous theorem which relates the obstruction to an extension and a certain functional cohomology operation. We apply this formula to extension problems where the range space is one of the classifying spaces $\mathrm{BU}, \mathrm{BO}$, or $\mathrm{BSp}$. Let $(X, A)$ be a $\mathrm{CW}$ pair and $f: A \rightarrow$ $Y$ a map with $Y_{f}$ the mapping cylinder of $f$. Assume that $\pi_{1}(Y)$ acts trivially on $\pi_{i}(Y)$ for all $i$. If $\eta \in H^{s+1}(G, r ; E)$ is a primary operation, it maps the cohomology sequence of the pair $\left(X \cup Y_{f}, Y_{f}\right)$ in dimension $r$ into the sequence in dimension $s+1$. This commutative diagram of groups defines a functional cohomology operation

$$
\Lambda_{f} \eta: H^{r}(Y ; G) \rightarrow H^{s+1}(X, A ; E)
$$

with indeterminacy equal to the image of $\eta$ plus the image of $\delta f^{*}$. We interpret the Hurewicz homomorphisms as a map $H^{s}(Y ; E) \rightarrow \operatorname{Hom}\left(\pi_{s}(Y), E\right)$.

Theorem 1. Let $(X, A)$ be a $C W$ pair and $f: A \rightarrow Y$ a map. Suppose that

Received by the editors March 12, 1974.

AMS (MOS) subject classifications (1970). Primary 55G35, 55G36; Secondary 55G37, 55G05, 55G45, 55F 45 .

Key words and phrases. Extension problems, obstructions, functional cohomology operations, Steenrod powers, Postnikov systems, the classifying spaces BU, $\mathrm{BO}$, and BSp. 
the extension obstruction $O^{s+1}(f)$ is nonvoid and that $\delta f^{*}$ has image zero in $H^{s+1}(X, A ; E)$. If $\eta \in H^{s+1}(G, r ; E)$ is a stable primary operation and $y \in H^{r}(Y ; G)$ is in the kernel of $\eta$, there is defined a set of homomorphisms, $\left\{f_{y \#}: \pi_{s}(Y) \rightarrow E\right\}$ which is a congruence class modulo the image of the Hurewicz homomorphism and has the following properties.

1. The set $f_{y^{*}} O^{s+1}(f)$ is independent of $f_{y \#}$ and $f_{y^{*}} O^{s+1}(f) \equiv \Lambda_{f} \eta(y)$ (mod image $\eta$ ).

2. If $[g] \epsilon \pi_{s}(Y)$, the functional operation $\eta_{g}(y)$ is defined and $\left\{f_{y \#}[g]\right.$ : $\left.f_{y \#}: \pi_{s}(Y) \rightarrow E\right\}=\eta_{g}(y)$.

In the next three theorems, $\delta_{p} P^{1}$ denotes the primary operation obtained by composing reduction $\bmod p$, the Steenrod power $P^{1}$, and the Bockstein $\delta_{p}$. We denote the Chern class in $H^{2 i}(\mathrm{BU} ; \mathrm{Z})$ by $c(i)$, the Pontryagin class in $H^{4 i}(\mathrm{BO} ; \mathrm{Z})$ by $p(i)$, and the symplectic class in $H^{4 i}(\mathrm{BSp} ; \mathrm{Z})$ by $y(i)$. In Theorem 3, $a_{n}=1$ if $n$ is even, 2 if $n$ is odd, and $a_{n} b_{n}=2$.

Theorem 2. Let $(X, A)$ be a $C W$ pair and $f: A \rightarrow \mathrm{BU}$ a map. Suppose that $O^{2 n+1}(f)$ is nonvoid and $\delta f^{*} c(n)=0$. For every prime $p \leq n-1$,

$$
p^{-1} n ! O^{2 n+1}(f) \equiv \Lambda_{f}\left(\delta_{p} P^{1}\right)(c(n-p+1))\left(\bmod \text { image } \delta_{p} P^{1}\right) .
$$

Theorem 3. Let $(X, A)$ be a $C W$ pair and $f: A \rightarrow \mathrm{BL}$ a map, where $\mathrm{L}=$ $O$ or Sp. Suppose that $O^{4 n+1}(f)$ is nonvoid and $\delta f^{*} p(n)=0$ if $\mathrm{L}=O$ or $\delta f^{*} y(n)=0$ if $\mathrm{L}=\mathrm{Sp}$. For every odd prime $p=2 k(p)+1$ such that $p \leq 2 n-1$,

$$
a_{n} p^{-1}(2 n) ! O^{4 n+1}(f) \equiv \Lambda_{f}\left(\delta_{p} P^{1}\right)(p(n-k(p)))\left(\bmod \text { image } \delta_{p} P^{1}\right),
$$

and

$$
b_{n} p^{-1}(2 n) ! O^{4 n+1}(f) \equiv \Lambda_{f}\left(\delta_{p} P^{1}\right)(y(n-k(p)))\left(\bmod \text { image } \delta_{p} P^{1}\right) .
$$

Theorem 4. Let $(X, A)$ be a $C W$ pair and $f: A \rightarrow \mathrm{BU}$ a map. Let the prime $p$ be the smallest torsion coefficient of the group $H^{*}(X, A ; Z)$ and suppose that for each group $H^{2 k+1}(X, A ; Z)$, all other torsion coefficients are strictly larger than $k-1$. If dimension $(X-A) \leq 2 n+2$ and $p \leq n-1<$ $2 p-1$, then $f$ extends over $X$ if and only if $\delta f^{*} c(i)=0,1 \leq i \leq n$, and

$$
0 \in \Lambda_{f}\left(\delta_{p} P^{1}\right)(c(j-p+1)), \quad p+1 \leq j \leq n .
$$

The relations between the above obstructions and the characteristic classes are as follows: 


$$
(n-1) ! O^{2 n+1}(f)=\delta f^{*} c(n), \quad a_{n}(2 n-1) ! O^{4 n+1}(f)=\delta f^{*} p(n),
$$

and

$$
b_{n}(2 n-1) ! O^{4 n+1}(f)=\delta f^{*} y(n) .
$$

(See [7, p. 95].) These formulas imply that if $H^{*}(X, A ; \mathbf{Z})$ is torsion free and $H^{*}\left(X, A ; \mathbf{Z}_{2}\right)=0$, homotopy classification and extension of mappings with range space $\mathrm{BU}, \mathrm{BO}$, or $\mathrm{BSp}$ can be accomplished with characteristic classes. (Also see [9]。) Theorems 2, 3, and 4 give some information on this type of homotopy classification or extension problem when $H^{*}(X, A ; \mathrm{Z})$ is not torsion free.

There are versions of the above theorems for the relative homotopy problem which can be obtained by replacing $\delta f^{*}$ by the difference homomorphism

$$
(f-g)^{*}: H^{r}(Y ; G) \rightarrow H^{r}(X, A ; G)
$$

and $\Lambda_{f} \eta$ by the corresponding homotopy operation

$$
\Lambda_{f, g} \eta: H^{r}(Y ; G) \rightarrow H^{s}(X, A ; E) .
$$

If $g$ is the constant map, $\Lambda_{f, g} \eta=\eta_{f}$. (See [4].) These theorems apply to the homotopy classification problem for maps from a lens space into a classifying space. If $L^{2 n+1}(m)$ is a generalized lens space with fundamental group $\mathbf{Z}_{m}$, let $p_{1}<p_{2}<\cdots<p_{t}$ be the prime divisors of $m$. We state theorems for maps from a lens space into $\mathrm{BU}$ or $\mathrm{BO}$. The corresponding $\mathrm{B} \mathcal{C}_{\mathrm{p}}$ theorem is obtained by replacing the condition $n \equiv 0(\bmod 4)$ in the BO theorem with the condition $n \notin 2(\bmod 4)$ and by replacing Pontryagin classes with symplectic classes.

Theorem 5. Let $f, g: L^{2 n+1}(m) \rightarrow \mathrm{BU}$ be a pair of maps and suppose that $p_{1}<p_{2}<\cdots<p_{s} \leq n-1<2 p_{1}-1$ for some $s$ such that $1 \leq s \leq t$. Then $f \cong g$ if and only if $f^{*} c(i)=g^{*} c(i), 1 \leq i \leq n$, and

$$
0 \in \Lambda_{f, g}\left(\delta_{p_{l}} P^{1}\right)\left(c\left(j-p_{l}+1\right)\right), \quad p_{l}+1 \leq j \leq n, \quad 1 \leq l \leq s .
$$

Theorem 6. Let $f, g: L^{2 n+1}(m) \rightarrow \mathrm{BO}$ be a pair of maps and suppose that $m$ is odd, $n \equiv 0(\bmod 4)$, and $p_{1}<p_{2}<\cdots<p_{s} \leq n-1<2 p_{1}-1$ for some $s$ such that $1 \leq s \leq t$. Then $f \cong g$ if and only if $f^{*} p(i)=g^{*} p(i), 1 \leq$ $i \leq p_{1}-1$, and 
$0 \in \Lambda_{f, g}\left(\delta_{p_{l}} P^{1}\right)\left(p\left(j-k\left(p_{l}\right)\right)\right), \quad k\left(p_{l}\right)+1 \leq j \leq p_{1}-1, \quad 1 \leq l \leq s$

2. Proofs of theorems. We sketch the proofs of the above theorems. Details of the proofs are found in [4]. The set of homomorphisms in Theorem 1 is obtained by using the operation $\eta$ to construct a two-stage Postnikov system $K(G, r ; E, s, \eta) \rightarrow K(G, r)$ with Eilenberg-MacLane invariant $\eta$. If $y: Y \rightarrow K(G, r)$, there are liftings of $y, f_{y}: Y \rightarrow K(G, r ; E, s, \eta)$, because $\eta(y)=0$ and the set of homomorphisms in the theorem is just the set of maps of homotopy groups induced by the liftings. The space $K(G, r ; E, s, \eta)$ is a loop space because $\eta$ is stable, and so it is easy to use the exact mapping sequence of the fibration $K(G, r ; E, s, \eta) \rightarrow K(G, r)$ to show that this set is a congruence class modulo the image of the Hurewicz homomorphism. The fact that $f_{y^{*}} O^{s+1}(f)$ is independent of $f_{y \#}$ follows from Olum's formula relating $O^{s+1}(f)$ and $\delta f^{*}$. (See [7, p. 84].) The formula

$$
f_{y^{*}} O^{s+1}(f) \equiv \Lambda_{f} \eta(y)(\bmod \text { image } \eta)
$$

means that $f_{y}, O^{s+1}(f)$ is contained in $\Lambda_{f} \eta(y)$. This follows from the fact that $\Lambda_{f} f \eta$ computes the second obstruction to extending the composite map $f_{y} f$ (see [6]), and standard naturality properties of obstructions and operations. The formula in property 2 is proved by using F. Peterson's definition of functional cohomology operations. (See [5, p. 157], or [10].)

Theorem 2 follows from Theorem 1. Because BU has no odd-dimensional cohomology, $\delta_{p} P^{1}(c(n-p+1))=0$, and so the set of homomorphisms $\left\{f_{n \#}^{p}\right.$ : $\left.\pi_{2 n}(\mathrm{BU}) \rightarrow \mathrm{Z}\right\}$ is defined. It follows from Bott's result on the Hurewicz homomorphism in $\mathrm{BU}$ (see [2] or [7, p. 93]) that this set is a congruence class modulo $(n-1)$ !, and we show that if $[g] \in \pi_{2 n}(B U)$ is a generator, then $f_{n \sharp}^{p}[g] \equiv p^{-1} n !(\bmod (n-1) !)$. This is established by using the second formula in Theorem 1 to show that

$$
f_{n \sharp}^{p}[g] \equiv\left(\delta_{p} P^{1}\right)_{g}(c(n-p+1))(\bmod (n-1) !) .
$$

This functional operation can be computed if we can compute

$$
\delta_{p} P^{1}: H^{2(n-p+1)}(g ; \mathbf{Z}) \rightarrow H^{2 n+1}(g ; \mathbf{Z}) .
$$

This is done with the aid of a formula of Serre and Borel on the action of $P^{1}$ on $H^{*}\left(\mathrm{BU} ; \mathrm{Z}_{p}\right)$ (see [1]), and a Hilton-Eckmann decomposition of BU having one cell for each homology generator which makes it possible to compute 
the Bockstein

$$
\delta_{p}: H^{2 n}\left(g ; \mathbf{Z}_{p}\right) \rightarrow H^{2 n+1}(g ; \mathbf{Z})
$$

Theorem 3 follows easily from Theorem 2 and well-known properties of the mappings $\mathrm{BL} \rightarrow \mathrm{BU}, \mathrm{L}=O$ or Sp. (See [3] or [7, p. 93].)

Theorem 4 is proved by showing that under hypotheses of the theorem, the containment formula in Theorem 2 is an actual equality in every relevant dimension. The proof uses a difference formula for obstructions due to Olum (see [8]) and F. Peterson's theorem on the orders of the $k$-invariants in the Postnikov system of BU. (See [9].) W. Singer's theorem on the divisibility of integral Chern classes (see [11]) is used to compute the orders of the images of the $k$-invariants in the cohomology of the Postnikov system of a connective fibering of BU. The se images act as higher order cohomology operations to compute the difference between obstructions in Olum's difference formula.

Theorems 5 and 6 follow from Theorems 2 and 3. In dimensions less than $2 n+1$, the cohomology of $L^{2 n+1}(m)$ with integer coefficients is $Z_{m}$ in even dimensions and 0 in odd dimensions, and so the characteristic classes do not determine the obstructions because of the presence of torsion, while our obstruction formulas are equalities with zero indeterminacy. In the real and symplectic cases, the extra obstruction in dimension $2 n+1$ is zero because $\pi_{2 n+1}(\mathrm{BO})=0$ if $n \neq 0(\bmod 4)$ and $\pi_{2 n+1}(\mathrm{BSp})=0$ if $n \neq 2(\bmod 4)$.

The results described in this note constitute part of the author's doctoral thesis [4]. I wish to thank Professor Paul Olum for his advice.

\section{REFERENCES}

1. A. Borel and J.-P. Serre, Groupes de Lie et puissances réduites de Steenrod dans la cohomologie des groupes classiques, Amer. J. Math. 75 (1953), 409448. MR 15, 338.

2. R. Bott, An application of the Morse theory to the topology of Lie-groups, Bull. Soc. Math. France 84 (1956), 251-281. MR 19, 291.

3. H. Cartan, Lecture notes, Séminaire Henri Cartan 12ième année: 1959/60, École Normale Supérieure, Secrétariat mathématique, Paris, 1961. MR 28 \#1092.

4. R. D. Little, A relation between obstructions and functional cohomology operations, Ph.D. Thesis, Cornell University, Ithaca, N. Y., 1972.

5. R. E. Mosher and M. C. Tangora, Cohomology operations and applications in homotopy theory, Harper and Row, New York, 1968. MR 37 \#2223.

6. P. Olum, Invariants for effective homotopy classification and extension of mappings, Mem. Amer. Math. Soc. No. 37 (1961). MR 23 \#A4144.

7. - Factorizations and induced homomorphisms, Advances in Math. 3 (1969), 72-100. MR $38 \# 6590$. 
8. P. Olum, Seminar in obstruction theory, Cornell Univ., Ithaca, N. Y., 1968.

9. F. P. Peterson, Some remarks on Chern classes, Ann. of Math. (2) 69 (1959), 414-420. MR $21 \# 1593$.

10. F. P. Peterson and N. Stein, Secondary cohomology operations: two formulas, Amer. J. Math. 81 (1959), 281-305. MR 23 \#A1366.

11. W. M. Singer, Connective fiberings over BU and U, Topology 7 (1968), 271303. MR $38 \# 717$.

DEPARTMENT OF MATHEMATICS, UNIVERSITY OF HAWAII, HONOLULU, HAWAII 96822 